\title{
COVID-19 X Violência: Qual a sua relação?
}

COVID-19 X Violence: What is your relationship?

COVID-19 X Violencia: ¿Cuál es tu relación?

Andrio Corrêa Barros

ORCID: https://orcid.org/0000-0002-2063-3177

Prefeitura Municipal de Primeira Cruz, Brasil

E-mail: andriobarros@hotmail.com

Geovanna Renaissa Ferreira Caldas

ORCID: https://orcid.org/0000-0001-9820-309X

Centro Universitário de Juazeiro do Norte, Brasil

E-mail: geovannacaldas@hotmail.com

Said Antonio Trabulsi Sobrinho

ORCID: https://orcid.org/0000-0002-0674-8672 UNICEUMA, Brasil

E-mail: saidtrabulsi@hotmail.com

Waiza Priscila Freire Oliveira

ORCID: https://orcid.org/0000-0003-0955-8951

Faculdade Pitágoras, Brasi

E-mail:waiza_priscila@hotmail.com

Liane Silva Sousa

ORCID: https://orcid.org/0000-0002-0194-7912

Faculdade Pitágoras, Brasil

E-mail: liasilsousaa@gmail.com

Ludmylle Rodrigues Silva França

ORCID: https://orcid.org/0000-0002-7719-9826

Prefeitura Municipal de Bom Jesus das Selvas, Brasil

E-mail: ludmyllefranca@gmail.com

Renata Porangaba Cavalcante

ORCID: https://orcid.org/0000-0002-9408-3251

UMJ- Centro Universitário Mario Pontes Juca, Brasil

E-mail: porangabarenata@gmail.com

Carla Carolina Alves Lopes

ORCID: https://orcid.org/0000-0002-8720-7738

Universidade Federal de São João Del Rei, Brasil

E-mail: carla.lopes01@outlook.com

Kelli Marise Alves da Silva Lopes

ORCID: https://orcid.org/0000-0002-8718-7968

Centro Universitário Una, Brasil

E-mail: kelli.lopes01@outlook.com

Camila da Costa Paula

ORCID: https://orcid.org/0000-0002-5752-6877

Universidade Federal de São João del Rei, Brasil

E-mail: mila.depaula1411@aluno.ufsj.edu.br

Larissa Cristina dos Santos Camargos

ORCID: https://orcid.org/0000-0003-1282-3074

Universidade de Rio Verde, Brasil

E-mail: larissacristinacamargos@hotmail.com

Isabela Santos Noivo

ORCID: https://orcid.org/0000-0002-0681-6321

Universidade de Rio Verde, Brasil

E-mail: isabela1noivo@gmail.com

Thallita Caroline Cassiano Gouvea

ORCID: https://orcid.org/0000-0002-6013-0128 Universidade de Rio Verde, Brasil

E-mail: thallita_gouvea@hotmail.com

Luann Morey Lemes

ORCID: https://orcid.org/0000-0002-0415-3085

Universidade de Rio Verde, Brasil

E-mail: drluannmorey@gmail.com

Juan Peron Faria Rocha

ORCID: https://orcid.org/0000-0002-2583-0989

Universidade de Rio Verde, Brasil

E-mail: peron.jjuan@gmail.com 
Renato Tavares Vieira de Oliveira

\begin{abstract}
Resumo
Objetivou-se evidenciar a relação direta entre o número de casos de violência e a pandemia da SARS-CoV-2. Trata-se de uma revisão integrativa da literatura, do tipo descritiva, através da busca via Biblioteca Virtual em Saúde, selecionando as bases de dados: Medical Literature Analysis and Retrieval Sistem Online, Literatura Latino-Americana e do Caribe em Ciências da Saúde e o Banco de Dados em Enfermagem - Bibliografia Brasileira, através da utilização dos Descritores em Ciências da Saúde: COVID-19, Violência e Pandemia, agregados ao operador booleano AND, entre os anos 2018 a 2020, selecionando assim, 09 artigos para confecção do estudo. Os casos de violência contra mulheres e crianças ocorrem frequentemente e a muito tempo, principalmente pela influência do patriarcado que está instaurado na sociedade, a pandemia e o isolamento social veio como um agravante perante toda essa situação. Conclui-se que em muitas pesquisas o número dos casos de violência apresentou-se inferior aos anos anteriores, mas que isso pode ter uma relação diretamente com o quanto os serviços e as ações de saúde foram afetados durante a pandemia do novo coronavírus.
\end{abstract}

Palavras-chave: COVID-19; Violência; Pandemia.

\begin{abstract}
The objective was to highlight the direct relationship between the number of cases of violence and the SARS-CoV-2 pandemic. This is an integrative literature review, of a descriptive type, through the search via the Virtual Health Library, selecting the databases: Medical Literature Analysis and Retrieval System Online, Latin American and Caribbean Literature in Health Sciences and the Nursing Database - Brazilian Bibliography, through the use of Health Sciences Descriptors: COVID-19, Violence and Pandemic, added to the Boolean operator AND, between the years 2018 to 2020 , thus selecting 09 articles for the preparation of the study. The cases of violence against women and children occur frequently and for a long time, mainly due to the influence of the patriarchy that is established in society, the pandemic and social isolation came as an aggravating factor in the face of this situation. It is concluded that in many studies the number of cases of violence was lower than in previous years, but that this may have a direct relation to how much health services and actions were affected during the pandemic of the new coronavirus.
\end{abstract}

Keywords: COVID-19; Violence; Pandemic.

\title{
Resumen
}

El objetivo fue resaltar la relación directa entre el número de casos de violencia y la pandemia SARS-CoV-2. Se trata de una revisión integradora de la literatura, de tipo descriptivo, a través de la búsqueda a través de la Biblioteca Virtual en Salud, seleccionando las bases de datos: Sistema de Análisis y Recuperación de Literatura Médica en Línea, Literatura Latinoamericana y Caribeña en Ciencias de la Salud y Base de Datos de Enfermería - Bibliografía Brasileña, utilizando Descriptores de Ciencias de la Salud: COVID-19, Violencia y Pandemia, agregado al operador booleano AND, entre los años 2018 a 2020, seleccionando así 09 artículos para el estudio. Los casos de violencia contra las mujeres y los niños ocurren con frecuencia y desde hace mucho tiempo, principalmente debido a la influencia del patriarcado que se establece en la sociedad, la pandemia y el aislamiento social vino como un factor agravante ante esta situación. Se concluye que en muchos estudios el número de casos de violencia fue menor que en años anteriores, pero que esto puede tener una relación directa con cuánto se vieron afectados los servicios y acciones de salud durante la pandemia del nuevo coronavirus.

Palabras clave: COVID-19; Violencia; Pandemia.

\section{Introdução}

No final do ano de 2019, na cidade de Wuhan, na China, surgiu um novo tipo de vírus de coronavírus, o SARS-CoV-2, causador da doença denominada de COVID-19. Essa doença espalhou-se rapidamente e intensamente por todo os países, a qual no final do mês de março do ano de 2020, a Organização Mundial de Saúde (OMS) declarou como uma pandemia (Cavalcante et al., 2020)

O Ministério da Saúde recomendou algumas medidas e alternativas não farmacológicas para um controle e enfrentamento da COVID-19, dentre elas, cita-se a higienização das mãos, uso de máscaras e o distanciamento/isolamento social (Brasil, 2020).

O distanciamento social provoca diversos sentimentos negativos na saúde psicológica da população, onde sensações de solidão passaram a ser comuns no dia a dia, incluídas com tristeza, alterações no sono e repouso, alterações no apetite, pavor de 
contrair a doença ou de algum ente querido contrair e pavor de não saber quando a situação irá melhorar. Além desses sentimentos, destaca-se os conflitos entre a família, ocasionados pela irritação, acrescida com o consumo exacerbado de drogas lícitas e ilícitas (Fornari et al., 2020).

Esses conflitos tem relação direta com o aumento dos casos de violência, Fornari et al (2021) confirma que durante a pandemia do novo coronavírus, houve um aumento de mais de $20 \%$ dos casos de feminicídios e aumento de mais de $37 \%$ do número de denúncias no mês de abril de 2020. A violência pode ser definida como quebra da integridade de uma pessoa, podendo ocorrer de forma física, mental, sexual, financeira ou moral, causando danos, sofrimentos até chegar ao óbito (Campos, Tchalekian \& Paiva, 2020).

Então, com a pandemia e as consequências que a mesma proporciona, como a crise financeira, social e comunitária, a interrupção dos trabalhos levando ao desemprego ou a empregos em home office, aumento do trabalho doméstico e fechamento e interrupções das escolas, isso fez com que as pessoas passassem mais tempo em sua residência. Esses fatores estão diretamente relacionados com o aumento do número de violências, pelo contato mais frequente da vítima com o agressor e pela diminuição do contato social da vítima com os seus entes queridos, dificultando o acesso a fontes de auxílio (Marques et al., 2020).

Além disso, outro ponto a ser considerado é a ausência das linhas de cuidados com a saúde, pois com o surgimento da COVID-19, a mesma passou a ser prioridade total, de certa forma, deixando mais de lado outros focos, como a saúde do idoso, da mulher e da criança, pois, segundo Oliveira, Duarte, França \& Garcia: "O país enfrenta não somente uma doença nova, mas também uma situação inusitada, que requer mudanças radicais de comportamento, nos níveis individual e comunitário" (Cruz et al., 2020).

Em decorrência disso, entende-se que o distanciamento social afeta gravemente a saúde da população, com alto risco de casos de violências. Sendo assim, surgiu-se a seguinte questão norteadora: Qual o impacto da pandemia da COVID-19 para a incidência dos casos de violência?

O presente estudo objetivou-se evidenciar a relação direta entre o número de casos de violência e a pandemia da COVID19.

\section{Metodologia}

O seguinte artigo trata-se de uma revisão integrativa da literatura, do tipo descritiva. Esse tipo de revisão tem grande relevância no sentido de analisar e interpretar dados prévios de outras fontes, através da realização de 04 etapas: Escolha dos descritores para a busca dos estudos, procurar estudos de fontes primárias, seguido de fontes secundárias e por fim, analisar, entender e sintetizar os dados obtidos (Bento, 2012).

Para obtenção dos dados e escrita do artigo, efetivou-se uma busca, entre os meses de abril e maio do ano de 2021,via Biblioteca Virtual em Saúde (BVS), selecionando as bases de dados: Medical Literature Analysis and Retrieval Sistem Online (MEDLINE), Literatura Latino-Americana e do Caribe em Ciências da Saúde (LILACS) e o Banco de Dados em Enfermagem - Bibliografia Brasileira (BDENF), através da utilização dos Descritores em Ciências da Saúde (DECS): COVID-19, Violência e Pandemia, agregados ao operador booleano AND, apresentando-se na seguinte sequência: "COVID-19 AND Violência AND Pandemia".

Em relação aos critérios de inclusão para selecionar os estudos que seriam utilizados, citam-se: artigos originais que estivessem disponíveis integralmente e de forma gratuita, publicados na língua inglesa ou portuguesa, divulgados entre os anos de 2018 a 2021 e que respondessem à questão norteadora, abordando a ideia central deste estudo. Entre os critérios definidos como de exclusão, citam-se: outras revisões, artigos repetidos, artigos baseados em cobertura de mídia e estudos que não respondessem à questão norteadora.

Para esclarecimento e melhor demonstração do método de busca e da seleção dos estudos utilizados, empregou-se um 
fluxograma baseado no protocolo PRISMA, delineado no Quadro 1.

Quadro 1 - Fluxograma de escolha dos estudos.

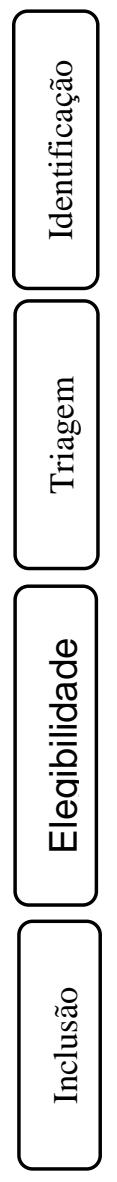

Ao realizar pesquisa dos

Decs na base de dados

MEDLINE ( $\mathrm{n}=150)$
Ao realizar pesquisa dos

Decs na base de dados

LILACS $(\mathrm{n}=35)$
Ao realizar pesquisa dos

Decs na base de dados

$\operatorname{BDENF}(\mathrm{n}=6)$
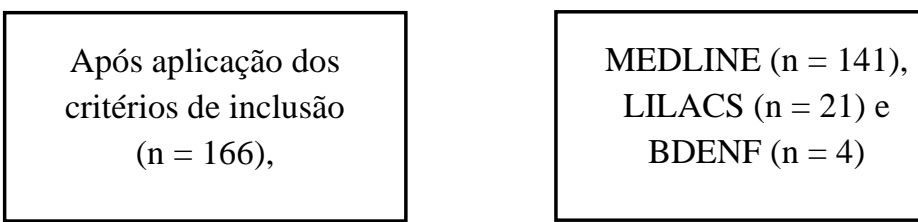
Artigos excluídos com base nos
critérios de exclusão definidos

$$
(\mathrm{n}=157)
$$

Estudos utilizados na pesquisa

$$
(\mathrm{n}=09)
$$

Fonte: Protocolo PRISMA

\section{Resultados}

Desses 09 estudos, foram extraídas informações para a montagem do quadro 02, incluindo: autores, ano, base de dados, idioma, título e periódico.

\begin{tabular}{|c|c|c|c|c|c|}
\hline $\mathbf{N}^{\mathbf{o}}$ & $\begin{array}{c}\text { AUTORES E } \\
\text { ANO }\end{array}$ & $\begin{array}{l}\text { BASE DE } \\
\text { DADOS }\end{array}$ & IDIOMA & TÍTULO & PERIÓDICO \\
\hline 01 & $\begin{array}{l}\text { Garstang, J., et al., } \\
2020 .\end{array}$ & MEDLINE & Inglês & $\begin{array}{l}\text { Effect of COVID-19 lockdown on child } \\
\text { protection medical assessments: a } \\
\text { retrospective observational study in } \\
\text { Birmingham, UK. }\end{array}$ & BMJ Open \\
\hline 02 & $\begin{array}{ll}\text { Campos, } & \text { B., } \\
\text { Tchalekian, } & \text { B., } \\
\text { Paiva, V., 2020. } & \end{array}$ & LILACS & Português & $\begin{array}{l}\text { Violência contra a mulher: } \\
\text { vulnerabilidade programática em } \\
\text { tempos de sars-cov-2/ covid-19 em são } \\
\text { paulo }\end{array}$ & Psicologia \& Sociedade \\
\hline 03 & $\begin{array}{l}\text { Vieira-Meyer, } \\
\text { A.P.G.F., et al., } \\
2021 .\end{array}$ & MEDLINE & Inglês & $\begin{array}{l}\text { Violence and vulnerability of the } \\
\text { Community Health Worker in the } \\
\text { territory: implications for tackling } \\
\text { COVID-19. }\end{array}$ & $\begin{array}{lll}\text { Ciência } & \& & \text { Saúde } \\
\text { Coletiva } & & \end{array}$ \\
\hline 04 & $\begin{array}{l}\text { Wong, J.Y-H., et } \\
\text { al., } 2021 .\end{array}$ & MEDLINE & Inglês & $\begin{array}{l}\text { Impact of COVID-19 on Child } \\
\text { Maltreatment: Income Instability and } \\
\text { Parenting Issues }\end{array}$ & $\begin{array}{l}\text { International Journal } \\
\text { Environmental Research } \\
\text { Public Health }\end{array}$ \\
\hline
\end{tabular}

Quadro 2 - Amostra dos artigos selecionados para compor o estudo. 


\begin{tabular}{|c|c|c|c|c|c|}
\hline 05 & $\begin{array}{l}\text { Ribeiro-Junior, } \\
\text { M.A.F., et al., } \\
\text { 2021. }\end{array}$ & MEDLINE & Português & $\begin{array}{l}\text { Estado atual do trauma e violência em } \\
\text { São Paulo - Brasil durante a pandemia de } \\
\text { COVID-19. }\end{array}$ & $\begin{array}{l}\text { Revista do } \text { Colégio } \\
\text { Brasileiro de Cirurgiões }\end{array}$ \\
\hline 06 & $\begin{array}{l}\text { Muldoon, K.A., et } \\
\text { al., } 2021 \text {. }\end{array}$ & MEDLINE & Inglês & $\begin{array}{l}\text { COVID-19 pandemic and violence: } \\
\text { rising risks and decreasing urgent care- } \\
\text { seeking for sexual assault and domestic } \\
\text { violence survivors. }\end{array}$ & BMC Medicine \\
\hline 07 & $\begin{array}{l}\text { Levandowski, } \\
\text { M.L., et al., } 2021 .\end{array}$ & MEDLINE & Português & $\begin{array}{l}\text { Impacto do distanciamento social nas } \\
\text { notificações de violência contra crianças } \\
\text { e adolescentes no Rio Grande do Sul, } \\
\text { Brasil. }\end{array}$ & $\begin{array}{l}\text { Cadernos de } \\
\text { Pública }\end{array}$ \\
\hline 08 & $\begin{array}{l}\text { Platta, } \\
\text { Guederta, } \\
\text { J.M., Coelho, } \\
\text { E.B.S., 2021. }\end{array}$ & MEDLINE & Português & $\begin{array}{l}\text { Violência contra crianças e } \\
\text { adolescentes: Notificações e alerta em } \\
\text { tempos de pandemia. }\end{array}$ & $\begin{array}{lll}\text { Revista } & \text { Paulista } & \text { de } \\
\text { Pediatria } & & \\
\end{array}$ \\
\hline 09 & $\begin{array}{l}\text { Katana, E., et al., } \\
2021 .\end{array}$ & MEDLINE & Inglês & $\begin{array}{l}\text { Violence and discrimination among } \\
\text { Ugandan residents during the COVID- } \\
19 \text { lockdown. }\end{array}$ & BMC Public Health \\
\hline
\end{tabular}

Fonte: Autores.

Dentre os artigos selecionados, 08 encontravam-se na MEDLINE e 01 na LILACS, sendo 05 na língua inglesa e 04 na língua portuguesa, com 02 publicados no ano de 2020 e 07 no ano de 2021.

\section{Discussão}

Observou-se nos seguintes estudos que a violência tem relação com a pandemia do novo coronavírus, isso ocorre devido ao estado de calamidade que o país se encontra, incluindo-se ainda pelo maior tempo dentro da residência, juntamente com o seu agressor.

Um estudo de Katana et al (2021) realizado com 1.726 pessoas apresentou um resultado de 167 casos de violência. Os casos de violência contra mulheres e crianças ocorrem frequentemente e a muito tempo, principalmente pela influência do patriarcado que está instaurado na sociedade, a pandemia e o isolamento social veio como um agravante perante toda essa situação, pois há um maior contato com o agressor, além do afeto ao ego masculino por não estar em domínio financeiro (Gomes, 2020).

Indo de contrapartida a isso, Wong et al (2021) refere que mais de 70 pais expuseram que a prevalência de violência física não alterou de forma significava e que em relação a violência psicológica, mais de 200 pais referem não haver ou notar mudanças durante a pandemia. Esses dados podem ter relações com a presença de homens como participantes do estudo, pois as mulheres são as principais afetadas nesses casos e também acabam sofrendo violência psicológica, ocorrendo naturalização de ofensas, temor de ir contra ao que o parceiro diz e a perspectiva dos homens serem os donos das mulheres (Souza \& Cassab, 2010).

Também indo contra esses resultados, Ribeiro-Junior et al (2021) evidenciam a incidência dos tipos de violência na cidade de São Paulo, citando-se a violência sexual, por arma de fogo, por arma branca e/ou autoprovocada. Muldoon et al (2021) confirmam esses dados, onde em sua pesquisa foi possível observar que ocorreu uma grande diminuição de mais de $56 \%$ das agressões sexuais e redução de mais de $48 \%$ das agressões físicas, considerando as pessoas que procuraram apoio e atendimentos nos serviços de saúde no período de tempo do estudo, seguindo esse resultado, Garstang et al (2020) observaram que houve redução de quase $40 \%$ da incidência desses casos, principalmente da referência escolar, pois como a instituição escolar não estava em seu completo funcionamento, perdeu-se um grande identificador dos sinais de violência e grande fonte de apoio para as vítimas.

Entretanto, a própria Organização Mundial de Saúde (OMS) evidencia que em situações de emergências, como uma pandemia do novo coronavírus, piora-se o quadro de saúde e aumenta o número de violência (Organização Mundial de Saúde, 
2021).

Esses resultados podem ter relação direta com a diminuição pela procura dos serviços essenciais de saúde, a Organização das Nações Unidas (ONU) confirmou que a pandemia de COVID-19 e a crise advinda da mesma, afetou-se drasticamente os serviços de saúde, de certa forma, deixando-se de lado outros problemas de saúde (Organização das Nações Unidas, 2020).

Legitimando essa hipótese para a diminuição dos casos de violência, todos os participantes da pesquisa, que eram profissionais da saúde, realizada por Campos, Tchalekian e Paiva (2020), referiram que houve grande redução da procura pelos serviços de saúde por mulheres, dificultando o processo de identificação de agressões e de criação de estratégias voltadas para essas vulnerabilidades e problemas de saúde.

Katana et al (2021) relata que de uma pesquisa com 1.726 pessoas, 779 não conseguiram ter algum tipo de acesso a serviços básicos, como alimentação e serviços de saúde. O acesso a saúde está diretamente relacionado com a oferta e disponibilidade dos serviços e sistemas, incluindo as desigualdades sociais e vulnerabilidades enfrentadas pela população. Ou seja, o grande número de casos de COVID-19 afetou os serviços de saúde, diminuindo a oferta para a população geral e com outras queixas, isso desencadeou uma menor busca de forma geral, incluindo-se as mulheres vítimas da violência (Stopa et al, 2017).

Confirmando esse mesmo aspecto, Marques et al (2020) referem que a pandemia reduziu o acesso aos serviços de saúde de maneira geral, mas afetando também as redes de apoio, como instituições escolares e instituições religiosas, as quais possuem um papel além do esperado, onde a população costuma enxergar como um local que pode ser buscado ajuda e/ou apoio. Netto et al (2017) também aborda as redes de apoio, dividindo-as em Primárias (outros familiares, vizinhos, amigos e colegas), Secundárias Formais (instituições de educação, saúde, segurança, justiça e de assistência social), Secundárias de Mercado (vínculo empregatício) e as Secundárias de Terceiro Setor (instituições religiosas).

Dentre os casos que foram notificados de violência, observa-se um padrão, sempre com predominância, das vítimas, no sexo feminino, maior em adolescentes e negras. É visível que as vulnerabilidades estão diretamente relacionadas com os casos de agressões, incluindo-se as vulnerabilidades sociais, econômicas e a desigualdade de gênero, que auxiliam no estigma da mulher ser inferior ao homem e depender dele em todos os sentidos, cabendo a ela sofrer tais consequências calada, sem direito a apoio. A anos que esse estigma existe sobre as mulheres e que busca mudanças positivas, porém com o isolamento social, todo esse quadro de situações foi gravemente intensificado, afetando a vida das mulheres ainda mais (Levandowski et al, 2021; Paz, Pires, Vieira \& Witt, 2019).

Considerando a área do infantojuvenil, Platt, Guedert e Coelho (2021) realizaram uma pesquisa no estado de Santa Catarina, por intermeio do Sistema de Informação de Agravos de Notificação (SINAN) e das Fichas de Notificação de Investigação Individual de Violência Interpessoal/Autoprovocada. Evidenciou-se mais de 1.800 notificações relacionadas a violência, seguindo a ordem numérica de maior quantidade: Negligência, Física, Sexual e Psicológica e com maior prevalência nas idades de 15 a 19 anos.

A residência da criança deveria, em teoria, ser o ambiente mais seguro possível, porém pelo alto nível de agressões pelos próprios familiares, acaba sendo um local de inseguranças e medos, constituindo o agressor e omissões pelos outros membros da família. Sendo assim, depara-se com uma maior exposição ao risco de agressões e um menor amparo, apoio e desenvolvimento de medidas voltadas para a proteção, devido ao distanciamento social (Platt, Guedert \& Coelho, 2021; Ferreira, 2020).

Em relação a essa temática estudada, segundo Maciazeki-Gomes, Souza, Baggio e Wachs (2016) os Agentes Comunitários de Saúde (ACS) possuem um papel importante na mediação entre a população e a equipe de saúde, sendo o primeiro contato que os usuários tem com a Estratégia de Saúde da Família (ESF), entretanto identificou-se que quase 70\% dos agentes não se consideravam capacitados para assistir os casos de violência na população. A importância do papel do ACS 
também é identificada nas relações entre os números das visitas domiciliares e os óbitos por COVID-19, quanto maior a abrangência do trabalho dos agentes, menores são as taxas de mortes pelo coronavírus (Vieira-Meyer, Morais, Campelo \& Guimarães, 2021)

Os profissionais da saúde desempenham um papel importantíssimo nessas situações, e conforme Oliveira et al (2018): “o profissional de saúde tem o dever de comunicar os casos de violência que tiver conhecimento, podendo responder por omissão". Essa relevância se destaca em todos os níveis de atenção, com destaque para a atenção primária e a possibilidade de atuar em conjunto com a prevenção, durante as consultas de planejamento familiar, pré-natal e puericultura, além do maior vínculo com a população (Oliveira, Duarte, França \& Garcia, 2020).

Como limitação para a escrita da pesquisa, cita-se a atualidade do assunto, ou seja, por ser um assunto muito novo, ainda não existem muitos estudos relacionados com a temática.

\section{Considerações Finais}

Conclui-se que em muitas pesquisas o número dos casos de violência apresentou-se inferior aos anos anteriores, mas que isso pode ter uma relação diretamente com o quanto os serviços e as ações de saúde foram afetados durante a pandemia do novo coronavírus. Essa pandemia gerou diversas consequências para e sobre o aspecto da saúde, dificultando o acesso da população e a criação e desenvolvimento de práticas voltadas para a prevenção e atuação sobre a violência contra as mulheres ou crianças.

Em decorrência da situação atual, a importância do profissional da saúde multiplicou-se, pois além de se preocupar e atentar a esse novo vírus, atuando ainda em todos os outros problemas de saúde, como a violência, pois a pandemia não os fez sumir, pelo contrário, a situação e os problemas só pioraram nessa época.

Como sugestões para trabalhos futuros, indica-se pesquisas realizadas para mulheres, para a identificação dos casos de agressão, sem contar apenas os notificados, pois sabe-se que a grande maioria sente medo de denunciar e o agressor não ser preso, além da dependência emocional e financeira.

\section{Referências}

Brasil, Ministério da Saúde (2020). Portaria No 1.565, de 18 de junho de 2020. Diário Oficial Da União, ed. 116, seção. 1, pág. 6. https://www.in.gov.br/web/dou//portaria-n-1.565-de-18-de-junho-de-2020-262408151.

Bento, A. (2012). Como fazer uma revisão da literatura: Considerações teóricas e práticas. Revista JA (Associação Académica da Universidade da Madeira), ${ }^{\circ}$ 65, ano VII: 42-44.

Campos, B., Tchalekian, B. \& Paiva, V. (2020). Violência contra a mulher: Vulnerabilidade programática em tempos de SARS-CoV-2/COVID-19 em São Paulo. Psicologia \& Sociedade, 32.

Cavalcante, J. R., et al. (2020). COVID-19 no Brasil: Evolução da epidemia até a semana epidemiológica 20 de 2020. Epidemiologia E Serviços de Saúde, 29(4).

Cruz, R. C. M. et al. (2020). Uma análise interprofissional do impacto do isolamento social na saúde da mulher durante a pandemia da COVID-19. Research, Society and Development, 9(11).

Ferreira, E. (2020). Uma pandemia em tempos de pandemia: o papel da escola no enfrentamento à violência sexual contra crianças e adolescentes. Enfrentamento ao abuso e à exploração sexual de crianças e adolescentes em tempos de pandemia. NCA-SGD, Boletim $\mathrm{n}^{\circ} 3$.

Fornari, L. F., Lourenço, R. G., Oliveira, R. N. G. de, Santos, D. L. A. dos, Menegatti, M. S. \& Fonseca, R. M. G. S. da. (2021). Domestic violence against women amidst the pandemic: coping strategies disseminated by digital media. Revista Brasileira de Enfermagem, 74(suppl 1).

Garstang, J., et al. (2020). Effect of COVID-19 lockdown on child protection medical assessments: a retrospective observational study in Birmingham, UK. BMJ Open, 10(9).

Gomes, K. S. (2020). Violência contra a mulher e Covid-19: dupla pandemia. Revista Espaço Acadêmico, 224.

Katana, E., et al. (2021). Violence and discrimination among Ugandan residents during the COVID-19 lockdown. BMC Public Health, 21(1).

Levandowski, M. L., et al. (2021). Impacto do distanciamento social nas notificações de violência contra crianças e adolescentes no Rio Grande do Sul, Brasil. Cadernos de Saúde Pública, 37(1). 
Research, Society and Development, v. 10, n. 7, e15510616246, 2021

(CC BY 4.0) | ISSN 2525-3409 | DOI: http://dx.doi.org/10.33448/rsd-v10i7.16246

Maciazeki-Gomes, R. de C., Souza, C. D. de, Baggio, L., \& Wachs, F. (2016). O trabalho do agente comunitário de saúde na perspectiva da educação popular em saúde: possibilidades e desafios. Ciência \& Saúde Coletiva, 21(5), 1637-1646.

Marques, E. S., et al. (2020). A violência contra mulheres, crianças e adolescentes em tempos de pandemia pela COVID-19: Panorama, motivações e formas de enfrentamento. Cadernos de Saúde Pública, 36(4).

Muldoon, K. A., et al. (2021). COVID-19 pandemic and violence: Rising risks and decreasing urgent care-seeking for sexual assault and domestic violence survivors. BMC Medicine, 19(1).

Netto, L. de A., et al. (2017). As Redes Sociais de Apoio às mulheres em situação de violência pelo parceiro íntimo. Texto \& Contexto - Enfermagem, 26(2).

Oliveira, B. G. de, et al. (2018). Responsabilidade dos profissionais de saúde na notificação dos casos de violência. Revista Bioética, 26(3), 403-411.

Oliveira, W. K. de, Duarte, E., França, G. V. A. de \& Garcia, L. P. (2020). Como o Brasil pode deter a COVID-19. Epidemiologia E Serviços de Saúde, Brasília, 29(2).

Organização Mundial de Saúde. Organização Pan-Americana da Saúde. (2021). Devastadoramente generalizada: 1 em cada 3 mulheres em todo o mundo sofre violência - OPAS/OMS. https://www.paho.org/pt/noticias/9-3-2021-devastadoramente-generalizada-1-em-cada-3-mulheres-em-todo-mundo-sofreviolencia\#: : :text=Next-,Devastadoramente\%20generalizada\%3A\%201\%20em\%20cada\%203\%20mulheres,todo\%20o\%20mundo\%20sofre\%20viol\%C3\%AA ncia\&text=\%E2\%80\%9CA\%20viol\%C3\%AAncia\%20contra\%20as\%20mulheres,\%2C\%20diretor\%2Dgeral\%20da\%20OMS.

Organização das Nações Unidas. (2020). Pandemia de Covid-19 causou severa redução de serviços básicos de saúde. ONU News. https://news.un.org/pt/story/2020/08/1721952.

Paz, P. de O., Pires, N.S., Vieira, L.B., \& Witt, R.R. (2019). Vulnerability of Women in Situation of Violence in Specialized Service. Aquichan, 19(2), Chia, Columbia.

Platt, V. B., Guedert, J. M., \& Coelho, E. B. S. (2021). Violence against children and adolescents: Notification and alert in times of pandemic. Revista Paulista de Pediatria, 39.

Ribeiro-Junior, M. A. F., et al. (2021). Current state of trauma and violence in São Paulo - Brazil during the COVID-19 pandemic. Revista Do Colégio Brasileiro de Cirurgiões, 48.

Souza, H. L de, \& Cassab, L. A. (2010). Feridas que não se curam: A violência psicológica cometida à mulher pelo companheiro. Anais do I Simpósio sobre Estudos de Gênero e Políticas Públicas - GT 5. Gênero e Violência, Universidade Estadual de Londrina, 24 e 25 de junho.

Stopa, S. R., et al. (2017). Use of and access to health services in Brazil, 2013 National Health Survey. Revista de Saúde Pública, 51(suppl 1).

Vieira-Meyer, A. P. G. F., Morais, A. P. P., Campelo, I. L. B., \& Guimarães, J. M. X. (2021). Violência e vulnerabilidade no território do Agente Comunitário de Saúde: Implicações no enfrentamento da COVID-19. Ciência \& Saúde Coletiva, 26(2), 657-668.

Wong, J. Y.-H., et al. (2021). Impact of COVID-19 on Child Maltreatment: Income Instability and Parenting Issues. International Journal of Environmental Research and Public Health, 18(4), 1501. 